\title{
EIGENVECTORS AND MAXIMAL VECTORS IN BOOLEAN VECTOR SPACES ${ }^{1}$
}

\begin{abstract}
RONALD L. SINZDAK
ABSTRACT. In this paper it is shown that every idempotent, selfadjoint linear endomorphism in a finite-dimensional normed Boolean vector space has its norm as an eigenvalue. A completely algebraic proof is also given for the fact that every linear endomorphism in such a space possesses a maximal vector.
\end{abstract}

1. Introduction. The concept of a normed Boolean vector space (or vector space over a Boolean algebra) was first introduced by N.V. Subrahmanyam in [3]. In [4], the same author defines a linear endomorphism of a Boolean vector space and P. V. Jagannadham [1] considered eigenvectors of such transformations.

It is the purpose of this paper to continue the study of eigenvectors and eigenvalues in normed Boolean vector spaces with finite bases. The main result is that every projection (idempotent, selfadjoint linear endomorphism) has its norm as an eigenvalue. The notion of a maximal vector is introduced and it is shown that every linear endomorphism has such a vector. It is also seen that a selfadjoint linear endomorphism with norm 1 has the property that the image of any maximal vector is a maximal vector.

2. Throughout this paper, $V$ is a normed Boolean vector space with a finite basis $G^{*}$ and $H$ is the set of all linear endomorphisms of $V$. Also, it is shown in [4] that for all $x$ in $V$ and $g$ in $G^{*}, x=\Sigma_{g}(x, g) g$.

Lemma 2.1. Every (nonzero) element of $V$ is an eigenvector of a linear endomorphism $T$ if and only if every element of $G^{*}$ is an eigenvector of $T$.

Proof. The necessity is clear.

For the sufficiency, suppose $\operatorname{Tg}=\lambda_{g} g$. Let $x \in V$ and let $\lambda=$

Received by the editors October 30, 1973.

AMS (MOS) subject classifications (1970). Primary 15A18.

Key words and phrases. Boolean vector space, eigenvectors, eigenvalues, adjoint, linear endomorphism, projection, maximal vector, normal endomorphism.

${ }^{1} A$ portion of this paper appears in the author's dissertation presented to the Graduate School of the University of Missouri and directed by Professor J. L. Zemmer. 
$\Sigma_{g}(x, g) \lambda_{g}$. Then

$$
\begin{aligned}
\lambda x & =\left(\sum_{g}(x, g) \lambda_{g}\right)\left(\sum_{g}(x, g) g\right)=\sum_{g}\left[\left(\sum_{g}(x, g) \lambda_{g}\right)(x, g)\right] g \\
& =\sum_{g}(x, g) \lambda_{g} g=\sum_{g}(x, g) T g=T\left(\sum_{g}(x, g) g\right)=T x .
\end{aligned}
$$

Thus, $x$ is an eigenvector of $T$. Note also that $\lambda=|T x|$ so that $|T x|$ is an eigenvalue of $T$.

Corollary. $T$ is a projection if and only if every (nonzero) element of $V$ is an eigenvector of $T$.

Proof. This follows from the above and [1, Theorem 10].

Theorem 2.1. Let $T \in H$ and let $A^{*}$ be the set of eigenvectors of $T$. Let $A$ be the set union of $A^{*}$ and $\{0\}$. Then $A$ is convex.

Proof. Let $x, y \in A$ and let $a \in B$. Let $w=a x+a^{\prime} y$. If $x=y=0$, then $w=0 \in A$. If $x=0$ and $y \in A^{*}$, then $w=a^{\prime} y$ where $T y=\beta y$ for some $\beta \in B$. Thus, $T w=T\left(a^{\prime} y\right)=a^{\prime} T y=a^{\prime} \beta y=\beta a^{\prime} y=\beta w$ so that $w \in A$. Similarly, if $y=0$ and $x \in A^{*}$, then $w \in A$.

Now suppose $x, y \in A^{*}$. Then $T x=\alpha x$ and $T y=\beta y$ for some $\alpha, \beta \epsilon$ $B$. Let $\lambda=a \alpha+a^{\prime} \beta$. Then

$$
\begin{aligned}
T w & =T\left(a x+a^{\prime} y\right)=a T x+a^{\prime} T y=a \alpha x+a^{\prime} \beta y \\
& =\left(a \alpha+a^{\prime} \beta\right)\left(a \alpha x+a^{\prime} \beta y\right)=\left(a \alpha+a^{\prime} \beta\right)\left(a x+a^{\prime} y\right)=\lambda w .
\end{aligned}
$$

Thus, if $w \neq 0$, then $w \in A^{*}$ and $A$ is convex.

Remark. It is easily shown that every linear transformation on a finitedimensional Euclidean space has an adjoint. Such is not the case in a Boolean vector space. The following lemma gives a necessary and sufficient condition for an adjoint to exist.

Lemma 2.2. Let $T \in H$. Then $T$ has an adjoint if and only if $(T g, T h)$ $=0$ for all $g, h \in G^{*}, g \neq h$.

Proof. If $g, h, k \in G^{*}$, and $T^{*}$ exists, then for $g \neq h,(T g, k)(T h, k)=$ $\left(g, T^{*} k\right)\left(h, T^{*} k\right)=0$ so that $(T g, T h)=\Sigma_{k}(T g, k)(T h, k)=0$.

Conversely, if $(T g, T h)=0$ for $g \neq h$, then $(T g, k)(T h, k)=0$ for $g \neq h$ and define $T^{*} k=\Sigma_{g}(T g, k) g$ so that $\left(T^{*} k, g\right)=(T g, k)=(k, T g)$ for all $g$, $h \in G^{*}$. Hence $T^{*}$ is the adjoint of $T$.

Corollary. If $T^{*}$ exists, then $|T|=\left|T^{*}\right|$. 
Proof. Since $|T|=\Sigma_{g}|T g|$ and $|T g|=\Sigma_{k}(T g, k)$ we have

$$
|T|=\sum_{g}\left(\sum_{k}(T g, k)\right)=\sum_{g}\left(\sum_{k}\left(g, T^{*} k\right)\right)=\sum_{g}\left|T^{*} g\right|=\left|T^{*}\right| .
$$

Terminology. If $T^{*}$ exists, we shall henceforth say that $T$ is adjointable.

Lemma 2.3. If $T$ is adjointable, then $T T^{*}$ and $T^{*} T$ are projections. Thus, if $T$ is selfadjoint, then $T^{2}$ is a projection.

Proof. By linearity, we have that for each $g \in G^{*}$,

$$
T T^{*} g=\sum_{k}\left[\sum_{b}\left(k, T^{*} g\right)(T k, b) b\right]=\sum_{k}\left[\sum_{b}(T k, g)(T k, b) b\right] .
$$

But $(T k, g)(T k, h)=0$ if $g \neq h$. Thus, $T T^{*} g=\left[\Sigma_{k}(T k, g)\right] g$ so that $T T^{*}$ is a projection.

Also, $T^{*} T g=\Sigma_{k}\left[\Sigma_{h}(T g, k)\left(T^{*} k, h\right) h\right]=\Sigma_{k}\left[\Sigma_{h}(T g, k)(T h, k) h\right]$. By Lemma 2.2, $(T g, k)(T h, k)=0$ if $g \neq h$. Thus, $T^{*} T g=\left[\Sigma_{k}(T g, k)\right] g$ and $T^{*} T$ is a projection.

Lemma 2.4. If $x, y \in V$, then $(x, y)<|x||y|$.

Proof. By the homogeneity of the inner product, $|x||y|(x, y)=$ $(|x| x,|y| y)=(x, y)$ so that $(x, y)<|x||y|$.

Lemma 2.5. Let $T \in H$ and $x \in V$. Then $|T x|<|T||x|$.

Proof. Since $(x, g)<|x|$ and $|T g|<|T|$,

$$
|T x|=\left|T\left(\sum_{g}(x, g) g\right)\right|=\left|\sum_{g}(x, g) T g\right|=\sum_{g}(x, g)|T g|<|x||T| \text {. }
$$

Theorem 2.2. If $T$ is adjointable and $x \in V, x \neq 0$, then $\left|T^{*} T x\right|=$ $|T x|$ and $x$ is an eigenvector of $T^{*} T$ with eigenvalue $|T x|$.

Proof. By Lemma 2.5, $\left|T^{*} T x\right|<\left|T^{*}\right||T x|$. Thus, $\left|T^{*} T x\right|<|T x|$. By the definition of inner product, $|T x|=(T x, T x)$, and by Lemma 2.4, $\left(T^{*} T x, x\right)<\left|T^{*} T x\right||x|$. Thus,

$$
|T x|=(T x, T x)=\left(T^{*} T x, x\right)<\left|T^{*} T x\right||x|<\left|T^{*} T x\right|
$$

and so $\left|T^{*} T x\right|=|T x|$.

It is shown in [2] that if $B_{x}$ is the set of all eigenvalues associated with $x$, then $B_{x}=\left\{\lambda \in B:|T x|<\lambda<|T x|+|x|^{\prime}\right\}$. It follows then from this, 
from Lemma 2.3, and from the above that $T^{*} T x=\left|T^{*} T x\right| x=|T x| x$.

Remark. It can be shown by induction that if $\left\{b_{1}, b_{2}, \cdots, b_{n}\right\}$ is a finite subset of $B$, then $b_{1}+b_{1}^{\prime} b_{2}+b_{1}^{\prime} b_{2}^{\prime} b_{3}+\cdots+b_{1}^{\prime} b_{2}^{\prime} \cdots b_{n-1}^{\prime} b_{n}=$ $\sum_{i=1}^{n} b_{i}$.

Definition. Let $T \in H$. An element $x$ in $V$ is called a maximal vector of $T$ if and only if $|x|=1$ and $|T x|=|T|$.

Theorem 2.3. Every linear endomorphism has a maximal vector.

Proof. Denote the elements of $G^{*}$ by $g_{1}, g_{2}, \cdots, g_{n}$. Let $T \in H$ and suppose $T g_{i}=\Sigma_{k=1}^{n} a_{i k} g_{k}$ where $a_{i p} a_{i m}=0$ if $p \neq m$. Let $b_{i}=\left|T g_{i}\right|=$ $\sum_{k=1}^{n} a_{i k}$. Let $a_{1}=b_{1}$ and for $1<i<n$, let $a_{i}=b_{1}^{\prime} b_{2}^{\prime} \cdots b_{i-1}^{\prime} b_{i}$. Finally, let $a_{n}=\left(a_{1}+a_{2}+\cdots+a_{n-1}\right)^{\prime}$. Note that if $i<n, a_{i}<b_{i}$. Clearly $a_{i} a_{n}=0$ for each $i \neq n$. Now suppose $i<n, j<n$ and $i \neq j$. Say $i<j$. Then $b_{i}^{\prime}$ appears as a factor in the representation of $a_{j}$. Thus, $a_{i} a_{j}<b_{i} b_{i}^{\prime}=0$ so that the " $a_{i}$ " are pairwise orthogonal.

Let $x=\sum_{i=1}^{n} a_{i} g_{i}$. Then $|x|=a_{1}+a_{2}+\cdots+a_{n}=a_{1}+a_{2}+\cdots+a_{n-1}$ $+\left(a_{1}+a_{2}+\cdots+a_{n-1}\right)^{\prime}=1$.

Now $T x=\sum_{i=1}^{n} a_{i} T g_{i}=\sum_{i=1}^{n} a_{i}\left(\sum_{k=1}^{n} a_{i k} g_{k}\right)$. By the orthogonality of coefficients, $|T x|=\sum_{k=1}^{n} \sum_{i=1}^{n} a_{i} a_{i k}$. But

$$
\sum_{k=1}^{n} \sum_{i=1}^{n} a_{i} a_{i k}=\sum_{i=1}^{n} a_{i}\left(\sum_{k=1}^{n} a_{i k}\right)=\sum_{i=1}^{n} a_{i} b_{i}
$$

By the definition of $a_{i}$ and by the preceding remark, it follows that $\sum_{i=1}^{n} a_{i} b_{i}=\sum_{i=1}^{n} b_{i}$. Hence, $|T x|=\sum_{i=1}^{n} b_{i}=\sum_{i=1}^{n}\left|T g_{i}\right|=|T|$ and $x$ is a maximal vector of $T$.

Remark. From Theorems 2.2 and 2.3 it follows that a selfadjoint linear endomorphism $T$ has a maximal vector $x$, which is an eigenvector of $T^{2}$ with eigenvalue $|T x|=|T|$. In particular, if $T$ is a projection, $|T|$ is an eigenvalue of $T$.

Theorem 2.4. Let $T$ be an adjointable linear endomorphism with maximal vector $x_{0}$. Let $y_{0}=|T| T x_{0}+|T|^{\prime} x_{0}$. Then $y_{0}$ is a maximal vector of $T^{*}$.

Proof. First, $\left|y_{0}\right|=|T|\left|T x_{0}\right|+|T|^{\prime}\left|x_{0}\right|=|T||T|+|T|^{\prime} 1=|T|+|T|^{\prime}=1$. Now $T^{*} y_{0}=|T| T^{*} T x_{0}+|T|^{\prime} T^{*} x_{0}=|T| T^{*} T x_{0}$ since $\left|T^{*} x_{0}\right|<\left|T^{*}\right|\left|x_{0}\right|=$ $|T|$ and hence $T^{*} y_{0}=|T|\left|T x_{0}\right| x_{0}=|T| x_{0}$. Thus, $\left|T^{*} y_{0}\right|=|T|=\left|T^{*}\right|$.

Remark. It follows from the above that if $T$ is an adjointable linear endomorphism with norm 1 and $x_{0}$ is a maximal vector of $T$, then $T x_{0}$ is a 
maximal vector of $T^{*}$. In addition, if $T$ is selfadjoint with norm 1 , then the image of any maximal vector of $T$ is a maximal vector of $T$.

Theorem 2.5. Let $T \in H$ and let $M$ be the set of all maximal vectors of $T$. Then $M$ is convex.

Proof. Let $x, y \in M$ and let $a \in B$. Let $w=a x+a^{\prime} y$. Then $|w|=$ $a|x|+a^{\prime}|y|$. But $|x|=|y|=1$ so that $|w|=a+a^{\prime}=1$. And since $T w=$ $a T x+a^{\prime} T y,|T w|=a|T x|+a^{\prime}|T y|$. But $|T x|=|T y|=|T|$ so that $|T w|=$ $a|T|+a^{\prime}|T|=|T|$. Thus, $w \in M$ and $M$ is convex.

Theorem 2.6. Let $T$ be a selfadjoint linear endomorphism with $|T|=1$. Let $M^{\prime}=\{\lambda x: \lambda \in B$ and $x \in M\}$. Let $S$ be the restriction of $T$ to $M^{\prime}$. Then $S$ is an isometry.

Proof. From the remark following Theorem 2.4, we have that if $x \in M$, $T^{2} x \in M$ so that $\left|T^{2} x\right|=|T|=1$. By Lemma $2.3, T^{2} x=\lambda x$ for some $\lambda \in B$. Thus, $1=\left|T^{2} x\right|=\lambda|x|=\lambda$. So $T^{2} x=x$ for any $x \in M$.

Now let $y \in M^{\prime}$, say $y=\lambda x$. Then $T^{2} y=T^{2}(\lambda x)=\lambda T^{2} x=\lambda x=y$. Thus, $T^{2} y=y$ for all $y \in M^{\prime}$ so that $S^{2}=I$ and $S$ is a bijection.

If $x, y \in M^{\prime}$, then $|x-y|=|S(S x)-S(S y)|<|S x-S y|<|x-y|$ and hence $|S x-S y|=|x-y|$.

Definition. Let $T$ be an adjointable linear endomorphism. Then $T$ is said to be normal if and only if $T T^{*}=T^{*} T$.

We now show that a vector which is an eigenvector of both $T$ and $T^{*}$ has the same eigenvalues with respect to both transformations. Further, if $T$ is normal, we show that $T$ and $T^{*}$ have the same eigenvectors.

Theorem 2.7. Let $T$ be adjointable and suppose $x$ is an eigenvector of both $T$ and $T^{*}$. Then $T x=T^{*} x$.

Proof. If $T x=\alpha x$ and $T^{*} x=\beta x$, then $\alpha|x|=(x, T x)=\left(T^{*} x, x\right)=\beta|x|$ and hence $a x=\beta x$.

Lemma 2.6. Let $T$ be an adjointable linear endomorphism. Then $T$ is normal if and only if $|T x|=\left|T^{*} x\right|$ for all $x$ in $V$.

Proof. If $T$ is normal, $|T x|=(T x, T x)=\left(x, T^{*} T x\right)=\left(x, T T^{*} x\right)=$ $\left(T^{*} x, T^{*} x\right)=\left|T^{*} x\right|$. Conversely, by Lemma 2.2 it follows that $\left(T T^{*} g, h\right)=$ $\left(T^{*} g, T^{*} h\right)=0$ and $\left(T^{*} T g, h\right)=(T g, T h)=0$ for $g \neq h$. For $g=h,\left(T T^{*} g, g\right)$ $=\left(T^{*} g, T^{*} g\right)=(T g, T g)=\left(T^{*} T g, g\right)$ by hypothesis. Hence $T T^{*}=T^{*} T$.

Corollary. If $T$ is normal, then $T$ and $T^{*}$ have the same maximal vectors. 
Proof. Let $x$ be a maximal vector of $T$. Then $|x|=1$ and $\left|T^{*}\right|=|T|=$ $|T x|=\left|T^{*} x\right|$. Thus, $x$ is a maximal vector of $T^{*}$. Similarly, any maximal vector of $T^{*}$ is a maximal vector of $T$.

Theorem 2.8. If $T$ is normal, then $T$ and $T^{*}$ have the same eigenvectors, and hence the same eigenvalues.

Proof. Let $x$ be an eigenvector of $T$. We know then that $T x=|T x| x$ so that $T^{*} T x=T^{*}(|T x| x)=|T x| T^{*} x$. By Lemma $2.3, T^{*} T x=\lambda x$ for some $\lambda \in B$. And since $T$ is normal, $|T x|=\left|T^{*} x\right|$ (Lemma 2.6). Thus, $\lambda x=|T x| T^{*} x=$ $\left|T^{*} x\right| T^{*} x=T^{*} x$ and so $x$ is an eigenvector of $T^{*}$. Similarly, every eigenvector of $T^{*}$ is an eigenvector of $T$ and it now follows from Theorem 2.7 that $T$ and $T^{*}$ have the same eigenvalues.

\section{BIBLIOGRAPHY}

1. P. V. Jagannadham, Linear transformations in a Boolean vector space, Math. Ann. 167 (1966), 240-247. MR 34 \#1239.

2. D. E. Rutherford, The eigenvalue problem for Boolean matrices, Proc. Roy. Soc. Edinburgh Sect. A 67 (1963/65), 25-38. MR 32 \#4056.

3. N. V. Subrahmanyam, Boolean vector spaces. I, Math. Z. 83 (1964), 422-433. MR $30 \# 43$.

4. - Boolean vector spaces. II, Math. Z. 87 (1965), 401-419. MR 30 $\# 3043$.

DEPARTMENT OF MATHEMATICS, UNIVERSITY OF SCRANTON, SCRANTON, PENNSYLVANIA 18510 